Received 00th January 20xx, Accepted 00th January 20xx DOI: $10.1039 / x 0 x \times 00000 x$ www.rsc.org/

\section{Soft modes of the dielectric response in the twist-bend nematic phase and identification of the transition to nematic splay bend phase in the CBC7CB dimer}

\author{
K. Merkel ${ }^{* a}$, A. Kocot ${ }^{a}$, C. Welch ${ }^{b}$ and G. H. Mehl ${ }^{b}$
}

${ }^{\text {a }}$ Faculty of Computer Science and Material Science, Institute of Technology and Mechatronics, University of Silesia in Katowice. Poland

${ }^{\mathrm{b}}$ Department of Chemistry, University of Hull, Hull HU6 7RX, UK

*katarzyna.merkel@us.edu.pl 


\section{Introduction}

In recent years, the has been a renewed interest in dimeric liquid crystal materials and bimesogens, mainly due to their extraordinary flexoelectric [1-3] and electro-optical properties [4-7] and for some dimeric materials the ability to form a modulated nematic phase $\left(\mathrm{N}_{\mathrm{TB}}\right)$ [8-14] has led to increased attention. In the $\mathrm{N}_{\text {тв }}$ phase, the director exhibits periodic twist and bend deformations forming a conical helix with doubly degenerate domains of opposite handedness $[3,15,16]$. The $\mathrm{N}_{\text {Tв }}$ phase in dimers was experimentally observed, mainly by a combination of experimental techniques such as polarized light microscopy, X-ray diffraction and Resonant Soft X-Ray Scattering (XRD, RSoXS) [5,9,11,17-21], light scattering [10] and freezefracture transmission electron microscopy (FFTEM) $[5,9,11]$. Additionally, the polarity and chirality of the $\mathrm{N}_{\text {тв }}$ phase formed by the achiral molecules has been investigated by electro-optical [4-7,12,22], magneto-optical [23], dielectric [22,24-27] and nuclear magnetic resonance studies (NMR) [28-31]. A number of theoretical models have attempted to describe the new nematic ground state and the anomalously low or negative bent elastic constants [32-35]. All these methods suggest a local helical structure of extremely tight pitch, in the region of 8 - $15 \mathrm{~nm}$ for dimers. Such nanoscale modulation of molecular orientation promises extremely short response times. Indeed the time of electro-optic switching reported for bent-core is about $1 \mu \mathrm{s}$ [25] and for $C B 7 C B$ and $C B 11 C B$ dimers even shorter times being in the sub-microsecond range have been detected, much shorter $[3,4,7]$ when compared to the millisecond range for uniaxial nematics. The field-induced distortional effect in the $\mathrm{N}_{\mathrm{TB}}$ phase may be extremely useful for technological applications as this has similarity to the electro-clinic effect $[4,35]$. In the $\mathrm{N}_{\text {Тв }}$ phase, the effect of an electric field can be observed through strong dielectric coupling as well as by a weak flexoelectric effect $\quad[2,37,38]$. A linear electro-optic effect is observed where the optical axis is rotated by the field applied in a plane perpendicular to the helical axis. Such a characteristic effect resembles the electroclinic effect, $(E C E)$, in the SmA* phase $[3,36]$ and flexo- electric effect (FEE) in the $N^{*}$ phase [39]. The analogy of $\mathrm{N}_{\text {тв }}$ phase to $\mathrm{N}^{*}$ phase is quite direct, as effects in both phases arise from flexo-electricity and both of these occur by distortions in the helical structure produced by the electric field. The analogy with the ECE in the chiral SmA phase $\left(\mathrm{SmA}^{*}\right)$ is much more subtle. It can be understood in terms of the generalized analogy between the pseudo-lamellar $\mathrm{N}_{\text {Тв }}$ phase and lamellar SmA*.

The main motivation of this paper is to analyze the molecular orientational dynamics of the $\mathrm{N}_{\mathrm{TB}}$ mesophase formed by the polar symmetric dimer $C B 7 C B$ and to study the behavior of this phase under an electric field. Dielectric spectroscopy under DC bias is used to investigate its response to an electric field. We observed the existence of soft modes due to the periodic structure of the $\mathrm{N}_{T B}$ phase and demonstrate that the electric distortions of the structure are not typically nematic-like but are very similar to those in the smectic and cholesteric phases as already shown by electrooptic [35] and light scattering [10,40]. Reviewing the latest dielectric research for the $C B 7 C B$ and related systems [22,26-28], we note that until now no one has reported the existence of a soft mode in LC dimers.

\section{Experimental}

The chemical structure of the 1"-,7"-bis(4cyanobiphenyl-4'-yl) heptane (CB7CB) is given in Figure 1a. The synthesis of the material has been reported recently $[4,41]$.

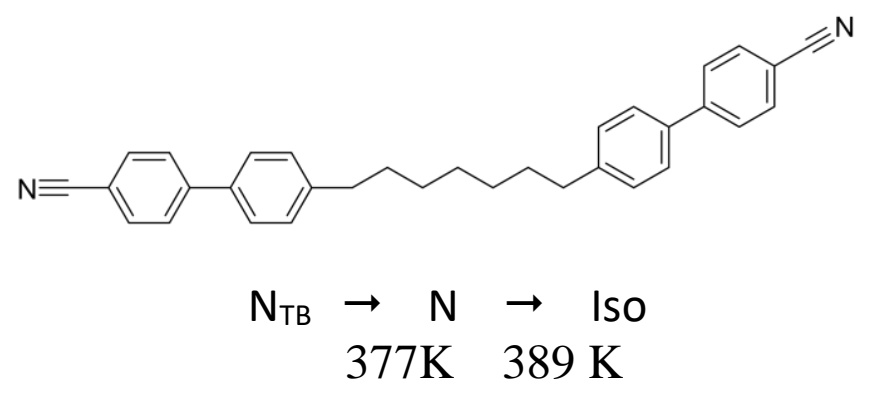

Fig.1. Chemical structure of the dimer $C B C 7 C B$ and transition temperatures [6]. 


\section{Dielectric measurements}

Dielectric spectroscopy studies were performed in the range from $0.1 \mathrm{~Hz}$ to $100 \mathrm{MHz}$ using two impedance/network analyzers: HP4I92A, HP4I95A and Solartron SI 1260 measured on a planar-aligned cell with and without the bias field. A number of cells with cell gaps varying from 1.6 to $12 \mu \mathrm{m}$ were tested. These include planar commercial cells with SE-130 polymer aligning (Nissan Chemical Industries, Ltd). The cells were assembled with antiparallel arrangement of the rubbing direction. The cell thickness was determined by using a spectrometer (Avaspec2048) interfaced with a PC. For the higher frequency dielectric measurements, gold plated cells were applied. The empty capacitance of the cell was measured before filling it with the material. The sample was then capillary filled by heating the empty cell in nematic phase, five degrees below the transition to the isotropic phase. Dielectric measurements were performed on slow cooling $0.1 \mathrm{~K} / \mathrm{min}$. Temperature of the sample was stabilized using PID temperature controller within $\pm 2 \mathrm{mK}$. Pt100 platinum resistor was used as a sensor and 4 points method was applied to compensate the leads resistance. Sample was placed in the cooper block in order to reduce the temperature gradient across the sample. For high-frequency range $(10-100 \mathrm{MHz})$ we use a parallel plate capacitor made of two circular gold-plated brass electrodes with $5 \mathrm{~mm}$ diameter separated by silica spacers. The sample is placed at the end of a coaxial line. The planar alignment in the range of the $\mathrm{N}$ and NTB phases was controlled by observing the amplitude of high frequency mode.

We presented dielectric results for planar $5 \mu \mathrm{m}$ sandwiched cells, the geometry of which is shown in the figure 2a.The amplitude of the probe field was in the range from 0.01 to $1 \mathrm{~V} / \mu \mathrm{m}$, whereas the $D C$ bias field up to $10 \mathrm{~V} / \mu \mathrm{m}$ was applied for studying the electric field dependence of the soft mode. The real $\left(\varepsilon^{\prime}\right)$ and imaginary $\left(\varepsilon^{\prime \prime}\right)$ parts of the complex permittivity were measured for planar cell under slow cooling from the isotropic state.

In order to determine the dielectric amplitude, $\delta \varepsilon_{\mathrm{j}}$, and the relaxation times, $\tau_{\mathrm{j}}$, of each relaxation mode, the dielectric spectra were analyzed using the Cole-Cole Eqn. (1) that expresses the complex permittivity in terms of the various relaxation processes:

$$
\varepsilon^{*}(\omega)-\varepsilon_{\infty}=\sum_{j=1}^{n} \frac{\delta \varepsilon_{j}}{\left[1+\left(i \omega \tau_{j}\right)^{1-\alpha}\right]},
$$

Here $\delta \varepsilon_{\mathrm{j}}, \tau_{\mathrm{j}}, \alpha_{\mathrm{j}}, \varepsilon_{\infty}$ are the fitting parameters of the equation to the experimental data of $\varepsilon^{\prime \prime}$. Results show that the high frequency dielectric spectra (frequency range above $0.1 \mathrm{MHz}$ ) are much more complicated than expected for the rigid core molecules. $\mathrm{CBnCB}$ molecules are highly flexible and we expected contributions to the spectra not only from rotations of the components of the total molecular dipole moment but also coming from rigid cores of the mesogens. The contributions of conformational changes to the $\varepsilon^{\prime \prime}$ spectra from parts of the mesogenic groups can also be detected.

For obtaining a better deconvolution of the relaxation peaks in the frequency range above $10^{5} \mathrm{~Hz}$, it is preferable to analyze the derivative of the real part of permittivity $\varepsilon^{\prime}$ [42] as given below:

$$
\frac{d \varepsilon^{\prime}}{d(\ln f)}=\frac{d \varepsilon^{\prime}}{d(\ln \omega)}=\sum_{j=1}^{n} \operatorname{Re} \frac{\delta \varepsilon_{j} \alpha\left(i \omega \tau_{j}\right)^{\alpha}}{\left[1+\left(i \omega \tau_{j}\right)^{\alpha}\right]^{2}},
$$

\section{Birefringence measurements}

The optical phase retardation of the material with and without the AC field was determined by the Sénarmont technique [43]. We used a microscope (Olympus BX56) equipped with a Sénarmont compensator (U-CSE, Leica) and a digital camera. The Sénarmont compensator consists of an accurate quarter-wave plate $(546 \mathrm{~nm})$ oriented so that one of its vibration directions, usually the high index (slow direction), is parallel to the polarizer vibration direction. We used a commercial in-plane switching cell (IPS) with planar alignment parallel to the electrodes (Fig. 2b). The IPS cell coated by SE-130 polymer has a cell gap of $5 \mu \mathrm{m}(\mathrm{d}=4.91 \mu \mathrm{m})$ and $40 \mu \mathrm{m}$ gap between electrodes. The geometry of birefringence measurements and the orientation of the thin IPS cell in the laboratory frame $x y z$ is shown in Fig.2b.

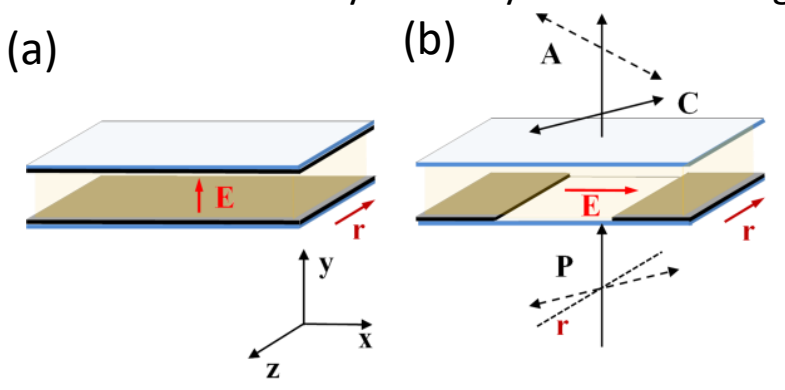


Fig.2. Geometry of planar cells in the laboratory frame xyz. (a) sandwich cell for dielectric measurements, (b) IPS cell for birefringence measurements. The rubbing direction, $\mathbf{r}$, parallel to the $\mathbf{z}$-axis, P-polarizer, $\mathbf{C}$ compensator, A-analyzer.

The birefringence can be calculated: $\Delta n=(\lambda / 2 \pi d)$ $\Delta \Phi$, were $\Phi$ is measured phase retardation and $d$ is a thickness of the cell.

We applied a $0.5 \mathrm{~Hz}$ AC field up to $10 \mathrm{~V} / \mu \mathrm{m}$ in the plane of the cell, perpendicular to the rubbing direction.

\section{Results and discussion}

\section{Dielectric properties}

Figure 3 shows the fitting examples of the $d \varepsilon^{\prime} / d$ $\log _{10} f$ at various temperatures $(374 \mathrm{~K}, 376 \mathrm{~K}, 378 \mathrm{~K}$ ) and how they reproduce the real, $\varepsilon^{\prime}$, and imaginary part, $\varepsilon^{\prime \prime}$, of the electric permittivity. It is clear that 2 peaks are dominant in the spectra of imaginary part, $\varepsilon$ ". Analyzing the temperature behavior of the $\mathrm{d} \varepsilon^{\prime} / \mathrm{d} \log _{10} f$ in the range below $376 \mathrm{~K}$ we can consider up to three relaxation peaks $(n \leq 3)$, in the frequency range above $0.1 \mathrm{MHz}$ and one in the low frequency range $\sim 10 \mathrm{~Hz}$. In the frequency range above $0.1 \mathrm{MHz}$ the various modes were obtained by fitting the experimental data to Eq. (2). The errors, are less than $7 \%$ for frequency and strength of the $\mathrm{MHz}$ peaks and $10 \%$ for frequency and $15 \%$ for strength of $0.1-1 \mathrm{MHz}$ peak. The shape parameters $\alpha$ were found quite small $(\alpha<0.07)$. Looking at Fig. 3 it is apparent that in the spectra of $\varepsilon^{\prime \prime}$ the third peak is not clearly visible. Figure 4a shows the $3-\mathrm{dm}$ plot of the derivative of electric permittivity, $\varepsilon^{\prime}$, vs. $\log _{10}$ of the frequency $\left(d \varepsilon^{\prime} / d \log _{10} f\right)$ in the frequency range $1 \mathrm{kHz}-100 \mathrm{MHz}$. The lowest frequency peak, was analyzed separately as it is not overlapping with the others.

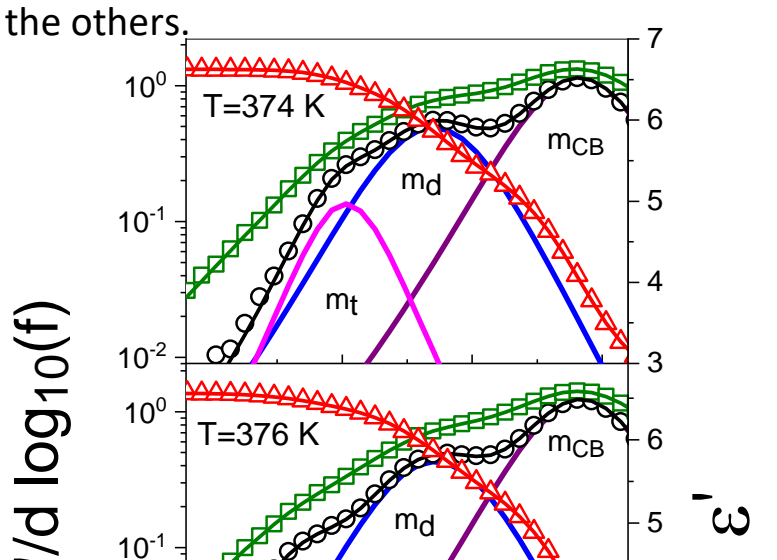

Fig.3. Frequency plots of the relative dielectric permittivity obtained for $5 \mu \mathrm{m}$ planar cell at different temperatures with respect to $(\log f)$. Symbols represent experimental points for: $\triangle$ - real part $\varepsilon^{\prime}, \square$ - imaginary part $\varepsilon^{\prime \prime}$ and $O$ - the derivative of $\varepsilon^{\prime}$. Solid lines are reproduced from fitting parameters. The violet, blue and magenta solid lines are the deconvoluted components of $-d \varepsilon^{\prime} / d\left(\log _{10} f\right)$ and correspond to the contributing modes: $\mathbf{m}_{\mathbf{C B}}, \mathbf{m}_{\mathbf{d}}, \mathbf{m}_{\mathbf{t}}$.

The Fig. $4 \mathrm{~b}$ shows the $3-\mathrm{dm}$ plot of imaginary part of the electric permittivity, $\varepsilon$ " in the frequency range $0.1 \mathrm{~Hz}-1 \mathrm{kHz}$. Conductivity was measured in the range of the $\mathrm{N}$ and the $\mathrm{N}_{\text {Tв }}$ phases. The conductivity was found about $1 \mathrm{nS} / \mathrm{m}$ in the $\mathrm{N}_{\text {TB }}$ and about $1.5 \mathrm{nS} / \mathrm{m}$ in the $\mathrm{N}$ phase. The temperature dependence is well described by $\sigma=\sigma_{0} \exp \left(-U / k_{\mathrm{B}} T\right)$ with $U=83.7 \mathrm{~kJ} / \mathrm{mol}$ and $\sigma_{0}=0.7 \mathrm{kS} / \mathrm{m}$. 
(a)

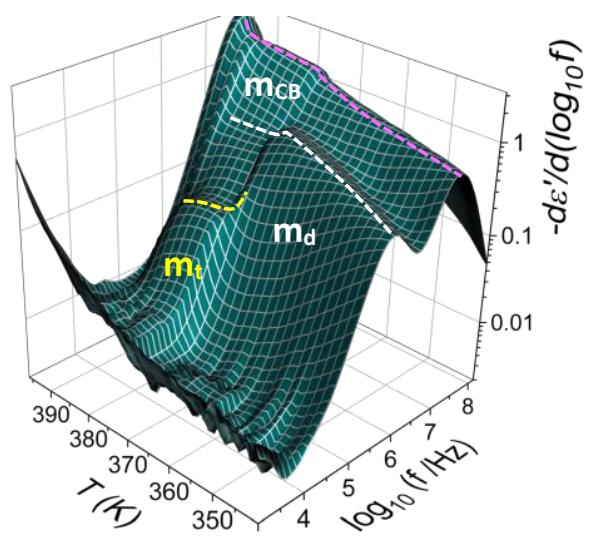

(b)

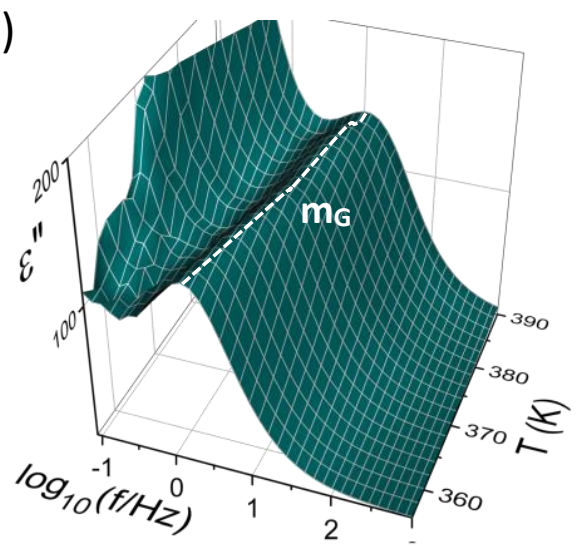

Fig.4. 3-D plot of the electric permittivity for $5 \mu \mathrm{m}$ planar cell as a function of the frequency and the temperature. (a) the derivative of the real permittivity, $\varepsilon^{\prime}$ vs. $\log _{10}$ of frequency $\left(-d \varepsilon^{\prime} / d\left(\log _{10}\right)\right)$ ), (b) the imaginary part of permittivity, $\varepsilon^{\prime \prime}$.

A set of relaxation frequencies was so obtained and we assigned two molecular modes $\mathrm{mCB}(\sim 70$ $\mathrm{MHz})$, md ( $\sim \mathrm{MHz})$ and two collective modes $\mathrm{mt}$ $(1 \mathrm{MHz})$ and $\mathrm{mG}(10 \mathrm{~Hz})$. These are plotted vs. temperature and this is shown in Fig. 5a. The corresponding relaxation frequencies, $f_{\mathrm{j}}=1 / 2 \pi \tau_{\mathrm{j}}$, are calculated from $\tau_{\mathrm{j}}$. From these frequencies the values for $\alpha$ are found to be in the range $0.05-0.07$. The corresponding amplitudes of the modes $\mathbf{m}_{\mathbf{c}}$, $\mathbf{m}_{\mathbf{d}}$ and $\mathbf{m}_{\mathbf{t}}$ are plotted in Figure $5 \mathrm{~b}$. For the temperature range above $376 \mathrm{~K}$, the spectrum is even more complex. The additional peak $\mathbf{m d}_{\mathbf{d}}$ which is due to the presence of other conformers can be deconvoluted. This peak can be assigned to the contribution of a hairpin-shaped conformer, likely to be present in the spectrum.

The amplitude of the highest frequency mode (mcB) was found to be a good probe of the biphenyl dipole moment distribution with respect to substrate normal. Due to monotonic decrease of the amplitude of the mode on lowering temperature (Fig.5), we conclude a gradual increase of the planar alignment on going from $\mathrm{N}$ to $\mathrm{N}_{\text {тв }}$ phase.
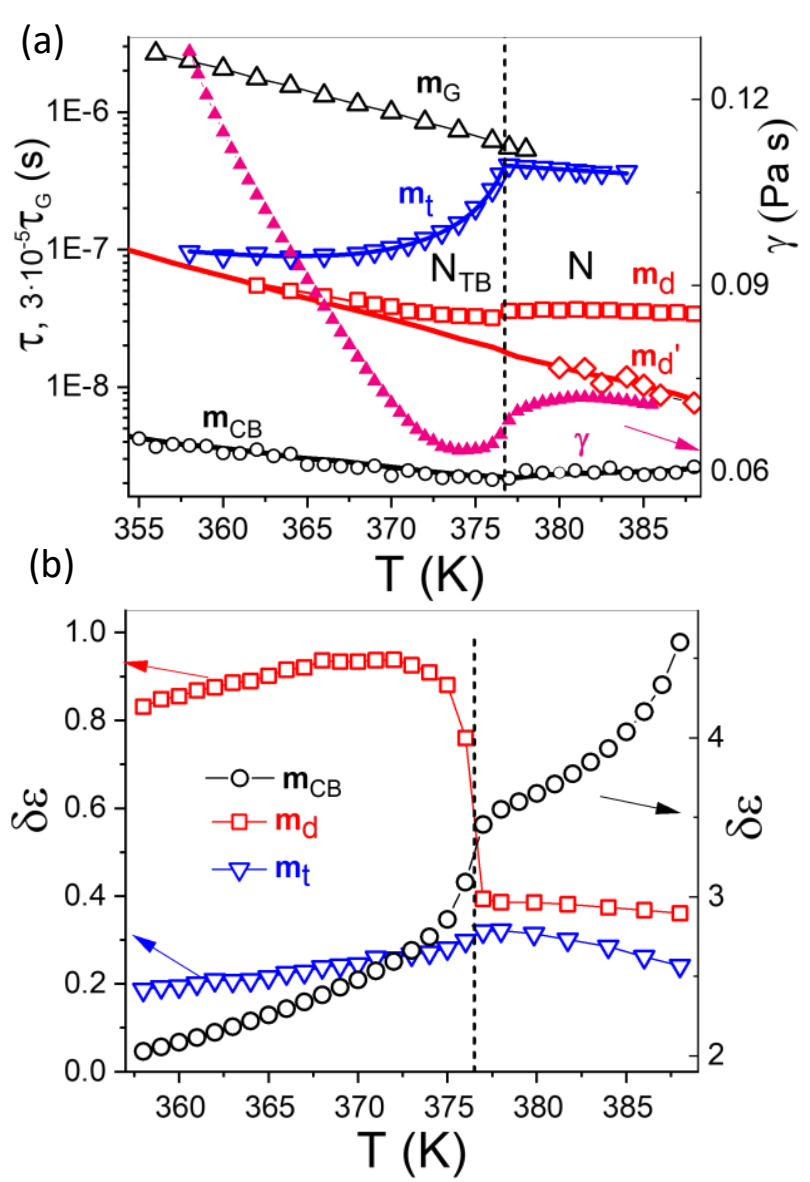

Fig. 5. (a) Plots of relaxation times for modes $(5 \mu \mathrm{m}$ planar cell): $\boldsymbol{\mu} \mathbf{1}$ - red line and $\boldsymbol{\mu} \mathbf{2}$ - black line, reported by López et al [25] and present results for modes: $\mathbf{m}_{\mathbf{C B}}-\mathrm{O}, \mathbf{m}_{\mathbf{d}}-\square, \mathbf{m}_{\mathbf{d}}$ '$\diamond, \mathbf{m}_{\mathbf{t}}-\nabla, \mathbf{m}_{\mathbf{G}}-\triangle\left(\mathbf{m}_{\mathbf{G}}\right.$ is multiplied by $\left.3 \cdot 10^{-5}\right)$, plotted as a function of temperature, a continuous blue line is a fit MF model to experimental data. The viscosity coefficient, $\gamma$ -

$\Delta$, calculated from relaxation time of the $m_{d}$ mode. (b)

Plots of the the dielectric amplitude $\delta \varepsilon$, of the modes mcB$\mathrm{O}, \mathbf{m}_{\mathbf{d}}-\square$, and $\mathbf{m}_{\mathbf{t}}-\nabla$ (is multiply by 2 ). 


\section{Molecular modes}

The complex permittivity expressed in terms of the Maier and Meier (M-M) model as given by Toriyama et al [44] is used here. The dynamics of the dielectric relaxation in the nematic phase is usually interpreted in terms of the rotational diffusion model for the reorientation of molecules in the nematic field, the model given by Coffey and Kalmykov [45]. The perpendicular component of the complex permittivity, $\varepsilon_{\perp}^{*}(\omega)$, can be written as:

$$
\varepsilon_{\perp}^{*}(\omega)-\varepsilon_{\perp \infty} \approx \frac{N^{\prime} h F^{2}}{3 \varepsilon_{0} k_{B} T}\left[\frac{\mu_{l}^{2}(1-S)}{1+i \omega \tau_{10}}+\frac{\mu_{t}^{2}\left(1+\frac{1}{2} S\right)}{1+i \omega \tau_{11}}\right]
$$

$A=N^{\prime} h F^{2} / 3 \varepsilon_{0} k_{B}$ is the scaling factor for the two relaxation mechanisms that contribute to the complex permittivity.

In Eq. (3), $N^{\prime}(=d / M)$ is the number density of molecules, $d$ is the mass density and $M$ is the molecular weight, $\varepsilon_{0}$ is the permittivity of vacuum, $T$ is the absolute temperature, $k_{B}$ is the Boltzmann constant. $F$ and $h$ are the internal field factors for the reaction and cavity fields, respectively. The $\mu_{1}$ and $\mu_{\mathrm{t}}$ are the longitudinal and the transverse projections of the molecular dipole moment directed along and normal to the long molecular axis, respectively. Thus, depending on the nature of the relaxation mode, the two terms on the righthand side of Eq. (3) relax at different frequencies, $f_{\mathrm{j}}=1 / 2 \pi \tau_{\mathrm{j}}$. In the rotational diffusion model [44], $\tau_{10}$ and $\tau_{11}$ are the relaxation times for the precessional and spinning rotations, respectively and are so assigned. Longitudinal, $\mu_{1}$, and transverse, $\mu_{\mathrm{t}}$, components of the molecular dipole moment, $\mu$, contribute to the dielectric permittivity differently and they relax at different frequencies of the probe field.

For dimers such analysis becomes much more complicated due to an existence of different conformers. In the case of high ordered dimers system when only two conformers: hairpin and bent-core are present the kinematic model by Stocchero et al. [46] is proposed. into discussion of molecular modes. This develops a theory for the dielectric relaxation of nematic dimers for which each of the mesogenic units is subjected to a nematic potential resulting in a four-state generalization of the Maier-Saupe potential.
Orientational relaxation occurs via individual endover- end reorientation of the rigid units and passage over a barrier of torsional potential for the carbon-carbon bonds of the alkyl chain. It should be noted here, that the flexible spacer imposes a correlation between both mesogenic units, accounted for by the equilibrium conformational distribution, which implies that the reorientations are considered to be individual but not independent. A major difference for it is that for dimers there are transitions amongst four stable states instead of two. Main conclusion that can be drawn is that: while the behavior of the parallel component significantly depends on the molecular structure the perpendicular component of the permittivity does not influence the temperature dependence on it [47]. In the present paper we analysed only the perpendicular component of the permittivity, $\varepsilon_{\perp}^{*}(\omega)$, thus the calculations for the rotational diffusion model are valid.

The corresponding relaxation peaks are found at the frequencies $f_{\mathrm{m} 2}=8 \mathrm{MHz}$ and $f_{\mathrm{m} 1}=70 \mathrm{MHz}$ (at 377 K) for transverse and longitudinal components respectively. The dielectric strengths of those two peaks, Fig.5a and their relaxation frequencies, Fig. $5 \mathrm{~b}$ are in good accordance with the earlier studies $[18,25,26]$ in the range of $N_{\text {tb }}$ phase. Additionally, we observed that the other peak, corresponding to the $\mathbf{m}_{\mathbf{t}}$ mode, contributes the relaxation spectra of $\varepsilon^{\prime \prime}$, at the frequencies of about $f_{\mathrm{m} 3}=1 \mathrm{MHz}$, an observation, which has not been reported before. This, however, cannot be subtracted without analyzing the derivative of $\varepsilon^{\prime}$.

In the $\mathrm{N}_{\text {Tв }}$ phase the contribution of the bent conformation is dominant. As the dimer is symmetric, the longitudinal component of its dipole is zero and the contribution to $\varepsilon_{\perp}$ is only due to the spinning rotation of the transverse component of the dimer dipole moment, thus it is assigned as a $\mathbf{m}_{\mathbf{d}}$, which is different than reported earlier assigned as a flip-flop motion CB unit, $\boldsymbol{\mu 1}$ (m1) $[18,25,26]$. The second relaxation, which must be due to precessional rotation of the longitudinal component, can arise only from a rotation of a part of the dimer, i.e. the cyanobiphenyl mesogenic core alone $\left(\mathbf{m}_{\mathbf{C B}}\right)$, in 
agreement agree with a mode $\boldsymbol{\mu} \mathbf{2}(\mathrm{m} \mathbf{2})$ from the data reported above. For the temperature range above $376 \mathrm{~K}$, the spectrum is even more complex. The additional peak $\mathbf{m}_{\mathbf{d}}$ ' which is due to the presence of other conformers can be observed. This peak can be assigned to the contribution of a hairpin-shaped conformer, likely to be present in the spectrum. The frequency of the spinning motion $\left(\mathbf{m}_{\mathbf{d}}\right)$ cannot be unambiguously established because of significant contributions of other conformers. This is reflected by an increase of the strength of transverse component, $\delta \varepsilon$, at the transition from the $N$ to $N_{\text {TB }}$ phase, where the contribution from the bent conformation becomes dominant. Unfortunately the accuracy of its frequency and strength are significantly reduced when both two modes: $m_{d}$ and $m_{d^{\prime}}$ are present. Thus in the uniaxial $\mathrm{N}$ phase, the results are quite complicated to analyze, as there are more conformers that contribute to the dielectric spectra. The reason for that is that the dynamics are strongly affected by conformational reorganizations of the molecules. In particular, an additional peak $\mathbf{m}_{\mathbf{d}}$ can be deconvoluted in the uniaxial $\mathrm{N}$ phase, which is due to the presence of other conformers, i.e. the hairpin-shaped conformer. On the transition from the $\mathrm{N}_{\text {тв }}$ phase to the $N$ phase a significant decrease of $\delta \varepsilon$ can be clearly seen, which is due to a reduction of the population of bent conformers (for other conformers the transverse component can be neglected). For the same reason the contribution of the $\mathbf{m}_{\mathbf{t}}$ mode becomes smaller, as it is related to longitudinal component of the dimer dipole moment. Another important reason for discrepancy between our results and those of $M$. Cestari et al [18], D. O. López et al [25] and RoblesHernández et al [26] is associated with the method of sample alignment. The relaxation time, for $\boldsymbol{\mu} \mathbf{1}$ mode is measured for homeotropic alignment but sample alignment has been achieved in this case by applying a strong electric field. This, however, is very likely to result in an increasing population of hairpin-shaped conformation in $N$ phase, which are expected to have a different dynamic behavior when compared with those have a bent core conformation. We note that in our case relaxation times of the $\mathrm{md}^{\prime}$ mode coincides with those reported by M. Cestari et al [18], D. O. López et al [25] and Robles-Hernández et al [26]).

At decreasing temperatures in the nematic phase, the dielectric anisotropy $\left(\triangle \varepsilon^{=} \varepsilon_{\|}-\varepsilon_{\perp}\right)$ becomes close to zero, and changes sign from positive to negative [26].

The dynamics for $\mathbf{m}_{\mathbf{d}}$ mode, i.e. its relaxation times are dependent on the order parameter, $S$. We can reproduce well the relaxation time $\tau_{11}$ by using, the parameter $S$, obtained from the amplitude of $\mathbf{m}_{\mathbf{~} \mathbf{B}}$ mode, see next paragraph. The relaxation time of the $\mathbf{m}_{\mathbf{d}}$ mode, on the other hand, can be related to rotational viscosity, $\gamma$, using formula:

$$
\tau=3 \gamma V \lambda / k_{B} T
$$

where $V$ is the volume per molecule of a LC dimer and $\lambda$, is the Perrin friction factor of asymmetric ellipsoid for its spinning rotation about the longest axis [48]. The factor, $\lambda$, was calculated to be 0.32 for the dimer molecule approximated by an ellipsoid with an axes ratio of 1:0.35:0.25. Thus both dynamic parameters, the viscosity and the diffusion coefficients (Fig.5) indicate an acceleration of the rotation on the transition to the $\mathrm{N}_{\text {TB }}$ phase.

\section{Collective modes}

The observed peak in the $\mathrm{MHz}$ frequency range, named $\mathbf{m}_{\mathbf{t}}$ mode, shows quite specific temperature behavior with a peculiarity at the $\mathrm{N}_{\text {TB }}$ to $\mathrm{N}$ transition. This is reminiscent of the soft mode at the transition from the SmA to the SmC phase, see Fig 6 . This peak can be assigned to a collective fluctuation of the tilt angle of the coarse grained director $\boldsymbol{N}$ out of the pseudo-layers normal, similar what has been reported for bent-core molecules [24]. As a response to an electric field one can expect electro-optic effects similar to the in-plane flexoelectric switching in the $\mathrm{N}^{*}$ phase. Such an electro-optic effect has been already observed for CB7CB dimer by Panov et al. [4] and C. Meyer et al 
[49]. A more extensive theoretical and experimental study by C. Meyer et al. [7] showed that this effect is also similar to the electroclinic effect (ECE) in the SmA* phase.

The $\mathrm{N}_{\text {TB }}$ and the cholesteric phases have some important common features: both phases are periodic and have a pseudo-layered structure, and both of them are chiral (the cholesteric due to the molecular chirality and the twist-bend nematic because of the structural chirality of the heliconical structure) [35]. Therefore, in all three phases ( $\mathrm{N}^{*}$, $\mathrm{SmA}^{*}$ and $\mathrm{N}_{\mathrm{TB}}$ ), when an electric field $\boldsymbol{E}$ is applied in the plane of the layers (or pseudo-layers), the optic axis rotates from its initial orientation, along the normal to the layers, to a new orientation, which lies in the plane of the layers. This effect is only possible in chiral systems, thus confirming the chiral symmetry of the $\mathrm{N}_{T B}$ phase even though it is formed by achiral molecules.

For the relaxation times of the $\mathrm{N}_{\text {тв }}$ distortion we obtain $[7,10,40]$ :

$$
\Gamma=1 / \tau_{t}=2 \pi f_{t}=\frac{D_{e f f}}{\gamma}
$$

where: $D_{\text {eff }} \approx \frac{1}{2}\left(K_{1}+K_{2}\right) q^{2} \sin ^{2} \theta$ is the effective elastic constant for the tilt in the coarse grained director $\boldsymbol{N}$ away from the pseudo-layer normal. $\boldsymbol{\gamma}$ is expected to be continuous at the $\mathrm{N}-\mathrm{N}_{\text {Tв }}$ transition temperature and $y$ is the rotational viscosity of the nematic phase. $D_{\text {eff }}$ is essentially linear in $\left(T_{\mathrm{TB}}-T\right)$. (Fig. 7), which confirms that the behavior of $D_{\text {eff }}$ is described by mean-field theory (M-F), except $4 \mathrm{~K}$ region just below $T_{\mathrm{TB}}$, where $D \propto\left(T_{\mathrm{TB}}-T\right)^{\times}$with $x \approx$ 0.67 follows more sophisticated, anisotropic scaling analysis [50].

We can try to reproduce the temperature dependence of the relaxation rate by using experimentally available parameters for the $C B 7 C B$ dimer in eq. (5). We used viscosity values obtained from the mode $\mathbf{m}_{\mathbf{d}}$, see Fig. $5 \mathrm{a}$, which is in good accordance with the bend viscosity in the range of $\mathrm{N}$ phase reported recently [43]. Wavenumber temperature dependence of the helix in the cell obtained from resonant carbon K-edge soft X-ray scattering by $\mathrm{C}$. Zhu et al [21] and the cone angle from our birefringence data measurements.
Reliable data for the elastic modulus are given for the CB7CB only in the range of $N$ phase $[43,51]$. Therefore $K_{\mathrm{t}}$ was used in eq. (5) as an unknown parameter in fitting the predicted relaxation rate to the values of experimental results.

The resulting elastic modulus is found to increase from 1 to $3 \mathrm{pN}$ in the range of the $\mathrm{N}_{T B}$ phase. It is assumed that the small measuring voltage $(0.1 \mathrm{~V} / \mu \mathrm{m})$ of the short period $(\sim \mu \mathrm{s})$ does not change the pitch and associated with that the wavenumber of the heliconical structure. The results of the model prediction are quite striking. The resulting temperature dependence of the elastic modulus shows a clear softening behavior at the transition temperature from the N to the NTB phase. We can compare the above results with the relaxation rate calculated from the electro-optic experiment reported by C. Meyer et al [49], see Fig. 6 . The electro-optic data initially shows a slight increase of the relaxation rate on decreasing temperature; then below $465 \mathrm{~K}$ the tendency reverses.

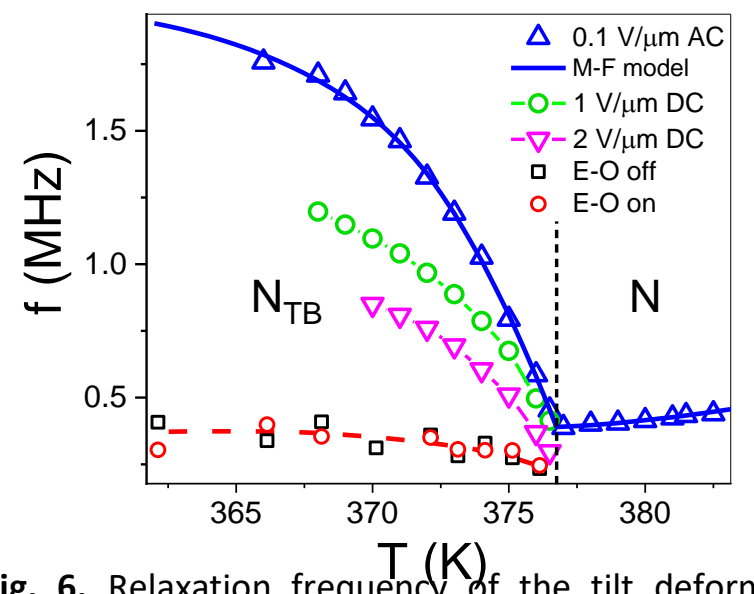

Fig. 6. Relaxation frequencl $\mathrm{K}_{\mathrm{f}}$ the tilt deformation mode, $\mathbf{m}_{\mathrm{t}}: f_{\mathrm{t}}-\Delta$. A continuous blue line is a fit of MF model to the experimental data. Relaxation frequencies of the helix tilt deformation mode under DC field $\mathrm{E}$ : $1 \mathrm{~V} / \mu \mathrm{m}-\mathrm{O}$, and $2 \mathrm{~V} / \mu \mathrm{m}-\nabla$. The temperature dependence of the relaxation frequency corresponding the electroclinic effect in the $\mathrm{N}_{\text {тв }}$ phase (square electric pulses with duration of $10 \mu \mathrm{s}, E=25 \mathrm{~V} / \mu \mathrm{m}$ ) [49], E-off$\square$ and E-on- $O$. 


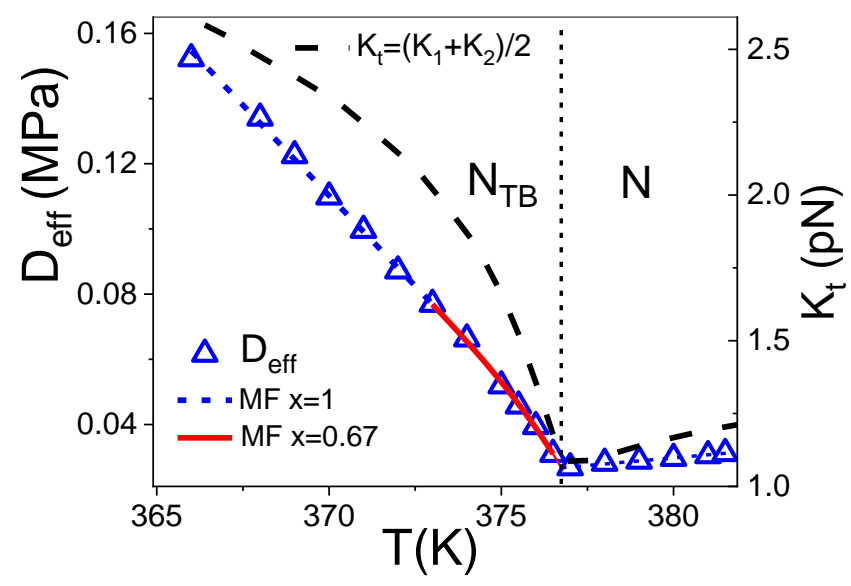

Fig. 7. Effective elastic constant for the tilt in the coarse grained director $\boldsymbol{N}-\Delta$. A dash blue line and continuous red line are fits of MF model to the experimental data of $D_{\text {eff. }} K_{\mathrm{t}}$ is elastic modulus obtained from the fitting, using eq.(5).

However there overall the curve is almost horizontal and shows a much slower relaxation rate $(\sim 0.4 \mathrm{MHz}$ ) than what we found from our relaxation data. The reason for this difference is intriguing. Indeed we note that the electro-optic experiment reported by $C$. Meyer et al [49] has been performed at a reasonably high electric field amplitude of $25 \mathrm{~V} / \mu \mathrm{m}$, nonetheless, the authors claim that the field pulse was sufficiently short $(10 \mu \mathrm{s})$ to consider the heliconical structure as conserved. We can test the strength of the influence of the bias field by applying a bias field (DC) in dielectric measurements. Fig. 6 shows the results for bias fields of $1 \mathrm{~V} / \mu \mathrm{m}$ and $2 \mathrm{~V} / \mu \mathrm{m}$.

With increasing the $D C$ field the curves are becoming gradually less steep, which would indicate an unwinding of the heliconical structure, i.e. diminishing the wavenumber of the helix in the cell. This corresponds to a pitch increase of about $20 \%$ and $36 \%$ for fields of $1 \mathrm{~V} / \mu \mathrm{m}$ and $2 \mathrm{~V} / \mu \mathrm{m}$ respectively. Thus, it is apparent that the field applied in earlier electro-optic experiments can significantly deform the heliconical structure. We note that for $\mathrm{N}_{\text {Тв }}$ forming dimers in the presence of high magnetic fields a strong deformation and change of transition temperatures has been reported too [52]. For bias fields this unwinding effect in the presence of strong fields occurs despite the short switching period of the applied field. We observe that a periodic field is less efficient than a
DC field; the corresponding pitch increases more than two times with respect to the off field helical structure.

The lowest frequency relaxation process, $\mathbf{m g}_{\mathbf{G}}$, is observed in the frequency range from $1 \mathrm{~Hz}$ to $10^{2}$ $\mathrm{Hz}$ (Fig. 4b). This process can be identified as a Goldstone mode, related to long scale phase fluctuations of the heliconical director $\mathbf{n}$, which does not change the coarse grained director $\boldsymbol{N}$ $[10,25,32]$. Such a long wavelength fluctuation mode should then be analogous to those found for the smectic-A phase (SmA), in particular the "slow", hydrodynamic layer compression.

In order to estimate the correlation length associated with fluctuations of the azimuthal angle of the local director we used a non-stationary solution of the Smoluchowski equation for angular coordinates, $\varphi$, (azimuthal angle) [53]. Physically, the diffusion corresponds to the change in director orientation $\Delta \varphi$ at distance $\Delta z \approx \Delta \varphi / q$ during the time $\Delta t=f^{-1}$. Thus, according to diffusion equation the estimation is: $\Delta z^{2} \approx D / q^{2} \omega$, where $D$ is the orientational diffusion coefficient. Inserting $D \approx 10^{9}$ $\mathrm{s}^{-1}, q=0.7710^{9} \mathrm{~m}$ and $\omega=50 \mathrm{~Hz}$ we estimated $\Delta z \approx 6$ $\mu \mathrm{m}$.

This mode should be described by an effective layer compression elastic constant $B_{\text {eff }}[10]$ (mainly associated with $K_{3}$ ) that plays the role of the elastic moduli for the compression of the pseudo-layer structure in the $\mathrm{N}_{\text {TB }}$ phase. The low frequency dielectric results confirm the "pseudo-layer" structure of the $\mathrm{N}_{\text {тв }}$ phase with an exceptionally low

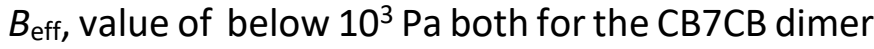
and in line what was earlier observed for bent core LCs [25] and is somewhat lower than what has been reported for difluoroterphenyl based dimers forming the $\mathrm{N}_{\text {тв }}$ phase [40]. The magnitude of $B_{\text {eff }}$ differs substantially from the typical value of $B_{\text {eff }} \cong$ $10^{6} \mathrm{~Pa}$ in a conventional SmA systems and that reported by E. Górecka et al [54] for a CB7CB sample.

When the cell thickness increases up to $7.1 \mu \mathrm{m}$ we observe the peak amplitude growing up to $\triangle \varepsilon \cong$ 900 , and saturates for $10 \mu \mathrm{m}$ and $12 \mu \mathrm{m}$. This is likely due to the ions screening effect, which becomes insignificant above $7 \mu \mathrm{m}$. The frequency of the peak decreases on the cell thickness increase as 
a result of the director undulation (flexoelectric effect) that accompanies the compression mode. We already measured the low frequency peak for two other homologs of $\mathrm{CBnCB}$ ( $n=9$ and 11) [55]. The peak are found to be present also in in the glassy state which, we think, rules out the space charge mobility as a main origin of the low frequency process.

It has to be noted, the field dependence of the mode frequency, Fig. 8, well follows the prediction of the theoretical model of the response of the bulk $N_{\text {тв }}$ phase to external electric fields within the framework of the Landau-de Gennes free energy expansion [33] in the similar way like we have already shown for BSMs [24]. Increasing the amplitude of the field up to $0.1 \mathrm{~V} / \mu \mathrm{m}$ does not change the mode frequency, but with a further increase of the field the mode frequency drops down significantly and then decreases almost linearly (Fig.8).

Assuming the mode frequency is proportional to $q^{2}$, $\left(B_{\text {eff }} \sim K_{3} q^{2} \sin ^{2} \theta\right)$ [35], the drop of the mode frequency at about $0.2 \mathrm{~V} / \mu \mathrm{m}$ coincides clearly with a jump of the wave number of the helical structure.

Following the model [33] the analysis of the data points towards the field induced formation the $\mathrm{N}_{S B}$ phase, generated at temperatures below the $\mathrm{N}$ phase stability in the presence of sufficiently high bias fields.

The lowest frequency mode $\left(m_{G}\right)$ may have other possibilities that can be considered as an origin of the mode. The existence of the low frequency mode has been already reported in the nematic phase of bent shape molecules both by light scattering Stojanovic et al [56], and dielectric relaxation $[24,57]$. G. Shanker et al [57] insist that close packing of orientationally ordered bent-shape molecules would tend to favors smectic layering on short length scales and appearance so-called smectic "cybotactic" clusters. That would lead to larger rotational viscosities and correspondingly slower orientational fluctuations. A continuous increase in mean cluster size can be expected on decreasing temperature and also on increasing electric field. This is, however, not observed in the experiment. Another concept of the mode origin is based on a dynamic light scattering study of a bent- core nematic phase. By exploiting the polarization selection rules of the scattering, it is possible to detected separate modes attributable to uniaxial director fluctuations and, in the lower part of the nematic phase, to fluctuations of the biaxial order parameter. The surprised fact is that the relaxation rate of the director modes in the bent-core material is roughly two orders of magnitude lower than the corresponding rate observed in typical straight core nematics. This is quite similar concept as suggested by Parsouzi et al [10] and Merkel et al [24], where the process is assigned to an azimuthal director fluctuations leading to compression of the pseudolayer structure in the $N_{\text {Тв }}$ phase combined with the undulation mode of the director.

It is intriguing, that the azimuthal director fluctuation (Goldstone mode) persists also in the nematic phase, despite the non-chiral nature of the nematic phase.

In fact chiral structures can arise also from achiral building blocks that lack a handedness if their assembly is unstable to chiral distortions, a phenomenon called spontaneous symmetry breaking. Chirality in LC requires transiently chiral molecules able to adopt chiral conformations, that can form macroscopic homo-chiral mono-domains, developing either from weak chiral perturbations or from random fluctuations.

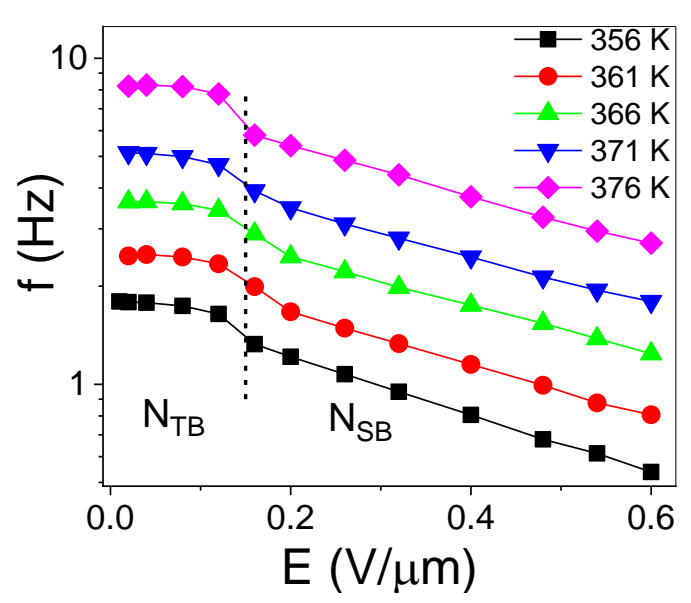

Fig.8. Frequency of the low frequency mode $f \sim q^{2}$ plotted as a function of $E$, for temperatures: $356 \mathrm{~K}-\mathbf{\square}, 361 \mathrm{~K}-\mathrm{O}$, $366 \mathrm{~K}-\triangle, 371 \mathrm{~K}-\nabla$ and $376 \mathrm{~K}-\nabla$. 
Link et al. [58] pointed out the chirality induced by tilting of BSMs. In the case of $\mathrm{CBnCB}(n=7,9,11)$ dimers we clearly observe chirality in $N_{\text {TB }}$ phase [55], which manifest itself by heliconical structure. However, it is noticeable deeply in nematic phase that molecules are tilted, which is indicated both by low order parameter (S ranged 0.4-0.55) and rather weak temperature dependence of the birefringence. This is usually accompanied by strong director fluctuation in the nematic phase that might be responsible for spontaneous symmetry breaking and the induced chirality. It was recently shown evidence of strong fluctuation of the twist bent deformation of the nematic director deeply in nematic phase far above the N-N transition [59]. This results in nonzero efficient value of the cone angle, which is usually observed by birefringence measurements.

\section{Phase structure rearrangement under E field}

In the $\mathrm{N}$ phase both amplitudes and relaxation frequencies of molecular modes can yield useful information about the dynamics of molecules and these can describe the orientational order of the $\mathrm{N}$ and $\mathrm{N}_{\text {тв }}$ phases. The amplitude of $\mathbf{m}_{\mathbf{d}}$ is clearly influenced by the conformational effects that can render information less evident. However, in the case of $\mathbf{m}_{\mathbf{c}}$ mode, the dipole moment of the cyanobiphenyl group lies on the average along the director and this can be an excellent probe for the orientational order parameter. We can obtain the order parameter $S$ by fitting the dielectric amplitude, $\delta \varepsilon$, of mcB mode in the isotropic and the $\mathrm{N}$ phase using

$$
\delta \varepsilon_{C B}=(1-S) \mu_{l}{ }^{2} N^{\prime} h F^{2} / 3 \varepsilon_{0} k_{B} T \text { as in eq.(3), }
$$

where: $B=\mu_{l}^{2} h F^{2} / 3 \varepsilon_{0} k_{B}$ is assumed to be a constant within the nematic phase. Thus $S$ can be obtained as: $S=1-\delta \varepsilon T / B N$ '. The result of this process is shown in the Fig. 9. The data can be fitted well in the range of the $\mathrm{N}$ phase using the formula:

$$
S=\left(1-T / T^{*}\right)^{\beta}
$$

where: $T^{*}$ is critical temperature that corresponds to the nematic-isotropic transition temperature $\left(T_{\mathrm{NI}}\right)$ and $\beta$ is the critical exponent coefficient. We found $T^{*}=389.2 \mathrm{~K}$ and $\beta=0.182$ as the best fit parameters. We are confident that $S$ describes how the director is distributed with respect to the substrate normal (i.e. y axis-insert in Fig. 9).

In a similar way birefringence measurements can be used to describe the distribution of the director in the substrate plane ( $x-z$ insert Fig.9). In the $\mathrm{N}$ phase the birefringence, $\Delta n$, has the usual behavior of a nematic, gradually increasing with decreasing temperature up to a value of 0.15 . The experimental data we obtained without field are in good accordance with earlier data $[12,45,60]$ in both nematic and $\mathrm{N}_{\text {TB }}$ phases. In the reported papers the data were fitted with the classical Haller formula [61]: $\Delta n=\Delta n_{0}\left(1-T / T_{N I}\right)^{\beta}$ using 3 parameters: $\Delta n_{0}, T_{N I}$ and $\beta$, where $\Delta n_{0}$ is the birefringence extrapolated to absolute zero, when the order parameter $S$ saturates to 1 .

We, however, found this formula over-described the birefringence $\Delta n$ as there are too many parameters for the fitting, and this influences the results. The values for the critical exponent reported in $[12,45]$ are too small, scattered and in our view less realistic than ours obtained from dielectric experiments $(\beta=0.182)$. Therefore we decided to use a fixed critical exponent $\beta=0.182$ and perform fitting of the $\Delta n$ data using only two parameters $\Delta \boldsymbol{n}_{0}$ and $T^{*}\left(\Delta \boldsymbol{n}_{0}=0.29\right.$ and $\left.T^{*}=389.2\right)$. Thus the order parameter, $S$, is simply obtained by rescaling $\Delta n$ by the factor 0.29 , Fig. 9 . The results from dielectric and birefringence are in good accordance within the range of $\mathrm{N}$ phase except a couple of degrees preceding the transition to the $\mathrm{N}_{\text {TB }}$ phase.

In the transition to $\mathrm{N}_{\text {тв }}$ phase, the heliconical structure is expected to transform the order parameter, $S$ into:

$$
S^{\prime}=S_{N} P_{2}(\cos \theta)
$$

where: $S_{\mathrm{N}}$ is the order parameter extrapolated from nematic phase, $P_{2}(\cos \theta)$ is second Legendre polynomial dependent on cosine of the cone angle $\theta$. The cone angle calculated from the formula is approaching $\theta=34^{\circ}$ at $T=356 \mathrm{~K}$, close to that observed for difluoroterphenyl dimers with a similar spacer using a combination of Se k-edge

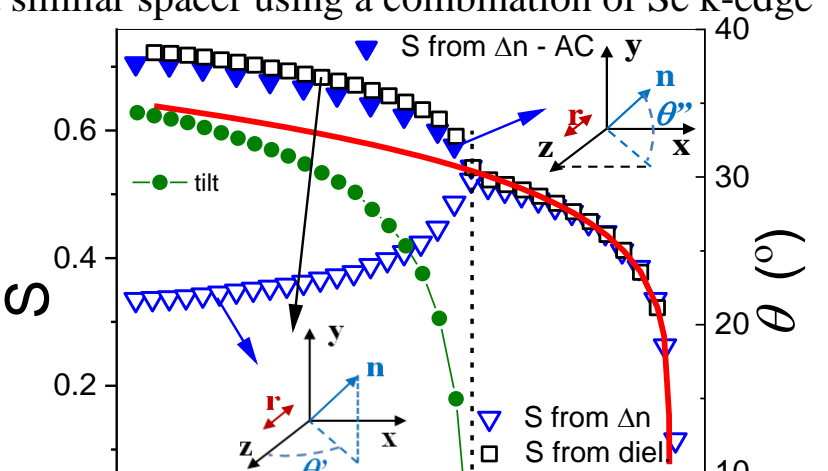


Fig.9. The orientational order parameter, $S$, obtained from the dielectric amplitude $\delta \varepsilon_{\mathrm{CB}}$ of mode $\mathrm{mCB}$ (precessional mode of the cyanobiphenyl dipole) $(5 \mu \mathrm{m}$ planar sandwich cell) plotted as a function of the temperature, $\square$ and its extrapolation due to eq.(7) shown by red solid line. $S$ calculated from $\Delta \boldsymbol{n}$ for planar sample $(5 \mu \mathrm{m}$ IPS cell) without field $-\nabla-, S$ calculated from $\Delta \boldsymbol{n}$ under the AC field $(5 \mathrm{~V} / \mu \mathrm{m})-\boldsymbol{\nabla}$-, Cone angle, $\theta$, from $\Delta \boldsymbol{n}$ measurements, - $\bullet$-.

scattering and GIXRD experiment [19].

The results for the cone angle obtained from the dielectric experiments are however quite different. On transition to the $\mathrm{N}_{\text {TB }}$ phase the parameter $S$ shows a significant increase despite our expectation to see a drop of the value of $S$ due to the formation of the heliconical structure. We are conscious that this effect can partly be a result of a more efficient molecular packing in $\mathrm{N}_{\mathrm{TB}}$ phase, when compared to the nematic phase. However, overall we accept that director is aligned within the substrate plane more closely than in the $\mathrm{N}$ phase, starting from $\theta^{\prime \prime}=10^{\circ}$ at the transition temperature and gradually approaching planar distribution $\left(\theta^{\prime \prime} 0^{\circ}\right)$ at $360 \mathrm{~K}$, while the distribution in the plane $(z-x)$ is gradually growing up to $\theta=34^{\circ}$, see Fig. 9 . The angles for vertical distribution are calculated from the order parameter, S, i.e. $\left\langle\cos ^{2} \theta^{\prime \prime}\right\rangle \cong 2 S / 3$ (assuming the dipole of the cyanobiphenyl groups makes the angle of $26^{\circ}$ with respect the dimer bow axis). We interpret the results as an indication of the formation of a surface induced biaxiality and then the formation of the nematic splay bend phase $\left(\mathrm{N}_{\mathrm{SB}}\right)$, in the $z-x$ plane. The effect is very similar to that observed for the de Vries SmA phase [62]. In the bulk SmA phase or in a homeotropic alignment a material showing the de Vries structure. A sample shows uniaxial conical distribution of the director. It has been shown that any type of external field such as an electric field or surface anchoring can destroy the uniaxiality and gradually increases phase biaxiality $[62,63]$.

In order to see the effect of the electric field on the $\mathrm{N}_{\text {Тв }}$ phase structure, we prepared the planar IPS sample ( $E$ in the plane of the substrate), Fig. $2 b$. By applying the electric field, that exceeds $E=4 \mathrm{~V} / \mathrm{mm}$, the birefringence and thus the order parameter can increase to the values larger than those extrapolated from the $\mathrm{N}$ phase; see Fig.9. The results are again indication of an electric field induced biaxial $\mathrm{N}_{\mathrm{TB}}$, which gradually develops into a nematic splay bend structure, $\mathrm{N}_{\mathrm{SB}}$, but now in the $\mathrm{z}$ y plane.

\section{Conclusion}

In summary; we observe four modes in the dielectric spectra of the material $C B C 7 C B$, the two higher frequency modes are assigned to the molecular modes and the other two are collective modes which are due to a distortion of the heliconical structure.

The collective mode in the $\mathrm{MHz}$ region is assigned to local distortions of the conical angle, while the periodic helical structure remains unaltered. The temperature dependence of the relaxation frequency of the mode and resulting elastic modulus have anomalous, softening-like behavior at the $\mathrm{N}-\mathrm{N}_{\text {TB }}$ transition. The dielectric method makes it possible to observe the modes without significant perturbation of the helical structure as opposed to the electro-optic methods. The lowest frequency collective mode, observed in the frequency range $1 \mathrm{~Hz}-10^{2} \mathrm{~Hz}$ is particularly interesting and it can be identified as a Goldstone mode, related to long-scale fluctuation of the cone phase. This results in an alternating compression and expansion of the pseudo-layer structure. We interpret the data of the measurements in the presence of strong bias fields in the temperature range where typically the $\mathrm{N}_{\text {TB }}$ phase is formed as an indication of the formation of a field induced nematic splay bend phase $\left(\mathrm{N}_{\mathrm{SB}}\right)$.

\section{Conflicts of interest}


There are no conflicts to declare.

\section{Acknowledgements}

Authors (KM \& AK) acknowledge funding by the National Science Centre, Poland, Grant No. 2018/31/B/ST3/03609. The author CW acknowledges funding by the EPSRC, grant EP/M015726/1. We thank Prof. I. Dozov for fruitful discussions. Authors (KM \& AK) thank Prof. W. Piecek, WAT, for supplying us with IPS cells.

\section{Notes and reference}

1. S. M. Morris, M. J. Clarke, A. E.Blatch, H. J.Coles, Phys. Rev. E, 2007, 75, 041701.

2. S. M.Shamid, S. Dhakal, J. V. Selinger, Phys. Rev. E, 2013, 87, 052503.

3. C. Meyer, G. R. Luckhurst, I.Dozov, Phys. Rev. Lett., 2013, 111, 067801.

4. V. P. Panov, R. Balachandran, J. K. Vij, M. G. Tamba, A. Kohlmeier, G. H. Mehl, Appl. Phys. Lett. 2011, 99, 261903.

5. D. Chen, J. H. Porada, J. B. Hooper, A. Klittnick, Y. Shen, M. R. Tuchband, E. Korblova, D. Bedrov, D. M. Walba, M. A. Glaser, J. E. Maclennan, N. A. Clark, Proc. Natl. Acad. Sci., 2013, 110, 15931.

6. V. P. Panov, J. K. Vij, G. H. Mehl, Liq. Cryst., 2017, 44, 147.

7. C. Meyer, Liq. Cryst., 2016, 43, 2144.

8. a) V.P. Panov, M. Nagaraj, A. Kocot, Y.P. Panarin, J. K. Vij, G. H. Mehl, 2010, P-3.36, 23rd ILCC, Kraków, b) V. P. Panov, M. Nagaraj, J. K. Vij, Y. P. Panarin, A. Kohlmeier, M. G. Tamba, R. A. Lewis, G. H. Mehl, Phys. Rev. Lett., 2010, 105, 167801.

9. V. Borshch, Y. K. Kim, J. Xiang, M. Gao, A. Jakli, V. P. Panov, J. K. Vij, C. T. Imrie, M. G. Tamba, G. H. Mehl, O. D. Lavrentovich, Nat. Comm. 2013, 4, 2635.

10. Z. Parsouzi, S. M. Shamid, V. Borshch, P. K. Challa, A. R. Baldwin, M. G. Tamba, C. Welch, G. H. Mehl, J. T. Gleeson, A. Jakli, O. D. Lavrentovich, D. W. Allender, J. V. Selinger, S. Sprunt, Phys. Rev. X, 2016, 6, 021041.

11. D. Chen, M. Nakata, R. Shao, M. R.Tuchband, M. Shuai, U. Baumeister, W. Weissflog, D. M. Walba, M. A. Glaser, J. E. Maclennan, N. A. Clark, Phys. Rev. E. 2014, 89, 022506.

12. C. Meyer, G. R. Luckhurst, I. Dozov, J. Mater. Chem. C, 2015, 3, 318.

13. J. P. Jokisaari, G. R. Luckhurst, B. A.Timimi, J. Zhu, H. Zimmermann, Liq. Cryst., 2015, 42, 708.

14. D. A. Paterson, R. Walker, J. P. Abberley, J. Forestier, W. T. A. Harrison, J. M. D. Storey, D. Pociecha, E. Gorecka, C.T. Imrie, Liq. Cryst., 2017, 44, 2060.

15. I. Dozov, EurPhys. Lett. 2001, 56, 247.

16. R. Memmer, Liq Cryst., 2002, 29, 483.

17. K. Adlem, M. Čopič, G. R. Luckhurst, A. Mertelj, O. Parri, R. M. Richardson, B. D. Snow, B. A. Timimi, R. P.Tuffin, D. Wilkes, Phys. Rev. E, 2013, 88, 022503.

18. M. Cestari, S. Diez-Berart, D. A. Dunmur, A. Ferrarini, M. R. de la Fuente, D. J. B. Jackson, D. O. Lopez, G. R. Luckhurst, M. A Perez-Jubindo, R. M. Richardson, J. Salud, B. A. Timimi, H. Zimmermann, Phys. Rev. E, 2011, 84, 031704
19. W. D. Stevenson, Z. Ahmed, X. B. Zeng, C. Welch, G. Ungar, G. H. Mehl, Phys. Chem. Chem. Phys., 2017, 19, 13449.

20. M. Salamończyk, N. Vaupotič, D. Pociecha, C. Wang, C. Zhu, E. Gorecka, Soft Matter., 2017, 13, 6694.

21. Ch. Zhu, M. R. Tuchband, A. Young, M. Shuai, A. Scarbrough, D. M. Walba, J. E. Maclennan, Ch. Wang, A. Hexemer, N. A. Clark, Phys. Rev. Lett., 2016, 116, 147803.

22. R. Balachandran, V. P. Panov, J. K. Vij, G. Shanker, C. Tschierske, K. Merkel, A. Kocot, Phys. Rev. E, 2014, 90, 032506

23. S. M. Salili, J. Xiang, H. Wang, Q. Li, D. A. Paterson, J. M. D. Storey, C. T. Imrie, O. D. Lavrentovich, S. N. Sprunt, J. T. Gleeson, A. Jákli, Phys. Rev. E, 2016, 94, 042705.

24. K. Merkel, A. Kocot, J. K. Vij, G. Shanker, Phys. Rev. E, 2018, 98, 022704.

25. D. O. López, N. Sebastian, M. R. de la Fuente, J. C. Martínez-García, J. Salud, M. A. Pérez-Jubindo, S. DiezBerart, D. A. Dunmur, G. R. Luckhurst, J. Chem. Phys. 2012, 137, 034502.

26. B. Robles-Hernández, N. Sebastián, M. R. de la Fuente, D. O. López, S. Diez-Berart, J. Salud, M. B. Ros, D. A. Dunmur, G. R. Luckhurst, B. A.Timimi, Phys. Rev. E, 2015, 92, 062505.

27. N. Sebastian, M. G. Tamba, R. Stannarius, M. R. de la Fuente, M. Salamonczyk, G. Cukrov, J.T. Gleeson, S. Sprunt, A. Jakli, C. Welch, Z. Ahmed, G. H. Mehl, A. Eremin, Phys. Chem. Chem. Phys., 2016, 18, 19299.

28. M. Cifelli, V. Domenici, S. V. Dvinskikh, G. R. Luckhurst, B. A. Timimi, Liq. Cryst., 2017, 44, 204.

29. J. W. Emsley, M. Lelli, A. Lesage, G. R. Luckhurst, J. Phys. Chem. B, 2013, 117, 6547.

30. A. Hoffmann, A. G. Vanakaras, A. Kohlmeier, G. H. Mehl, D. J. Photinos, Soft Matter, 2015, 11, 850.

31. J. P. Jokisaari, G. R. Luckhurst, B. A. Timimi, J. Zhu, H. Zimmermann, Liq. Cryst., 2015, 42, 708.

32. E. I. Kats, V. V. Lebedev,. JETP Lett., 2014, 100, 110.

33. G. Pająk, L. Longa, Chrzanowska, Proc. Natl. Acad. Sci., 2018, 115, E10303.

34. M. A. Osipov, G. Pająk, Liq. Cryst., 2017, 44, 58.

35. C. Meyer, I. Dozov, Soft Matter, 2016, 12, 574.

36. S. Garoff, R. B. Meyer, Phys Rev. Lett., 1977, 38, 848.

37. H. J. Deuling, Mol. Cryst. Liq. Cryst., 1972, 19, 123.

38. N. Vaupotič, M. Čepič, M. A. Osipov, E. Gorecka, Phys. Rev. E, 2014, 89, 030501(R).

39. J. S. Patel, R. B. Meyer, Phys. Rev. Lett., 1987, 58, 1538.

40. Z. Parsouzi, A. S. Pardaev, C. Welch, Z. Ahmed, G. H. Mehl, A. R. Baldwin, J. T. Gleeson, O. D. Lavrentovich, D. W. Allender, J. V. Selinger, A. Jakli and S. Sprunt, Phys. Chem. Chem Phys., 2016, 18, 31645.

41. K. Krzyżewska, T. Jaroch, A. Maranda-Niedbała, D. Pociecha, E. Górecka, Z. Ahmed, C. Welch, G. H Mehl, A. Proń, R. Nowakowski, Nanoscale, 2018, 10,16201.

42. K. Merkel, A. Kocot, J.K. Vij, G. Mehl, T. Mayer, Phys. Rev. $E, 2006,73,051702$. 
43. G. Babakhanova, Z. Parsouzi, S. Paladugu, H. Wang, Yu. A. Nastishin, S. V. Shiyanovskii, S. Sprunt, O. D. Lavrentovich, Phys. Rev. E, 2017, 96, 062704.

44. H. Toriyama, S. Sugimari, K. Moriya, D. A. Dunmur, R. Hanson, J. Phys. Chem. 1996, 100, 307.

45. W.T. Coffey, Y. P. Kalmykov, Advances in Liquid Crystals: A Special Volume of Advances in Chemical Physics, edited by J. K. Vij (John Wiley \& Sons, 2000), Vol. 113.

46. M. Stocchero, A. Ferrarini, G.J. Moro, D.A. Dunmur, G.R. Luckhurst, J. Chem. Phys., 2004, 121 (16), 8079.

47. N. Sebastián, B. Robles-Hernández, S. Diez-Berart, J. Salud, G.R. Luckhurst, D.A. Dunmur, D.O. López, M.R. de la Fuente, Liq. Cryst., 2017, 44 (1), 177.

48. a) G. K. Youngren, A. Acrivos, J. Chem. Phys., 1975, 63, 3846. b) R. J. Sension, R. M. Hochstrasser, J. Chem. Phys., 1993, 9, 2490.

49. C. Meyer, I. Dozov, P. Davidson, G. R. Luckhurst, I. Dokli, A. Knezevic, A. Lesac, XIII, Proc. SPIE. 2018, $105550 Z$ (8 February 2018); doi: 10.1117/12.2301296.

50. P.-G. de Gennes and J. Prost, The Physics of Liquid Crystals, 2nd ed. (Oxford, New York, 1993), Chapters 6 and 10.

51. S Parthasarathi, D. S. S. Rao, N. B. Palakurthy, C.V. Yelamaggad, S. K. Prasad, J. Phys. Chem. B, 2016 _ 120, (22), 5056.

52. S M. Salili, M. G. Tamba, S. N. Sprunt, C. Welch, G. H. Mehl, A. Jákli and J. T. Gleeson, Phys. Rev Lett., 2016, 116, 217801.

53. S. A. Pikin, in Relaxation Phenomena in Dielectric Magnetic and Superconducting Materials, Springer-Verlag, Heidelberg Berlin, (2003).

54. E. Górecka, N. Vaupotic ${ }^{2}$, A. Zep, D. Pociecha, J. Yoshioka, J. Yamamoto, and H. Takezoe, Angew. Chem. Int. Ed. 2015, 54, 10155

55. K. Merkel, C. Welch, Z. Ahmed, W. Piecek, G. H. Mehl, J. Chem. Phys. 2019, 151, 114908.

56. S. Stojadinovic, A. Adorjan, and S. Sprunt, H. Sawade, A. Jakli, Phys. Rev. E, 66, 2002, 060701 R.

57. G. Shanker, M. Nagaraj, A. Kocot, J. K. Vij, M. Prehm, C. Tschierske, Adv. Funct. Mater., 2012, 22, 1671.

58. D. R. Link, G. Natale, R. Shao, J. E. Maclennan, N. A. Clark, E. Korblova, D. M. Walba, Science, 1997, 278, 1924.

59. D. Pociecha, C. A. Crawford, D. A. Paterson, J. M. D. Storey, C. T. Imrie, N. Vaupotic, E. Gorecka, Phys. Rev. E, 2018, 98, 052706.

60. C. Meyer, C. Blanc, G. R. Luckhurst, I. Dozov, The $13^{\text {-th }}$ European Conference on Liquid Crystals, Sep 2015, Manchester, U.K. Domain Soft Condensed Matter.

61. I. Haller, Prog. Solid. State. Chem., 1975, 10, 103.

62. A. Kocot, T. S. Perova, K. Merkel, J. K. Vij, V. Swaminathan, S. P. Sreenilayam, N. Yadav, V. P. Panov, P. J. Stevenson, A. Panov and D. Rodriguez, J Chem. Phys. 2017, 147, 094903.

63. M. Nagaraj, K. Merkel, J. K. Vij, A. Kocot, Europhys. Lett. 2010, 91, 66002. 